\title{
GMPLS Energy Efficiency Scheme for Green Photonic Networks
}

\author{
M. Nazri M. Warip, Ivan Glesk, Ivan Andonovic \\ EEE Department, University of Strathclyde, 204 George Street, Glasgow G1 1XW, United Kingdom \\ Tel: (44141) 548 2529, Fax: (44141) 552 4968,e-mail: ivan.glesk@eee.strath.ac.uk
}

\begin{abstract}
ABSTRATCT
Since its emergence the internet has been a significant part of today's modern living. Defined by its interconnections and routing policies, it has fuelled increased demands for provisioning of new more advanced services that are able to dynamically react to changes within the network. These services however, lead to enormous energy consumption in contrast to a global drive for a greener environment. Hence the existence of an optical infrastructure that complies with the principles of zero-carbon emission is imperative.

Subsequently, in this paper, we present an energy model of Generalized Multi-Protocol Label Switching (GMPLS) network for more power efficient Green Photonic Networks. We are proposing a greener network design based on a novel routing algorithm to deliver power reduction through implementation of so called "Hibernation" approach. The scheme includes network topology such as group the nodes configuration, segmentation of the link/ports, and wavelength provisioning via partitioning. The performance evaluations of these energy saving schemes are investigated by including various challenging issue on "greening the internet" and reduces carbon footprint. In addition, to study the impact of wavelength request, blocking probability and power consumption in relation to network load is taken into account. A trade-off is observed between energy per bit, wavelengths offered (Erlang) and blocking probability as a result of the idling nodes.
\end{abstract}

Keywords: GMPLS, Green Photonic Networks, power consumption, energy efficiency.

\section{INTRODUCTION}

The internet has been the vital part of our modern ways of living with consumers constantly demanding fastsuper-efficient services. Previously, however, such demands for better services often resulted in power consumption increases which in turn led to putting the environment at greater risk.

The green photonic networks needs are often seen as a pathway leading to trigger new inventions and innovations, offering more energy awareness and healthier lifestyle for everyone. The contribution of photonics is considered green if [1]: (1) Generates or helps to conserves energy; (2) Reduces greenhouse gases emissions; (3) Reduces pollution; (4) Leads to improved public health.

In consideration of all the above, this paper emphasis is on the reduction of power consumption and energy efficiency optimisation in optical networks by implementing novel routing algorithm in Generalized MultiProtocol Label Switching (GMPLS) control plane. Application of this methodology results in significant reduction of energy usage as well as cutting down the optical core network operating cost without compromising connectivity. The issues and challenges arising in development of greener photonic networks and the evolution of the implementation of a proper control plane will also be discussed. However, optical technologies leading to energy savings in optical networks also needs very serious consideration.

\section{NETWORK ENERGY MODEL}

The network energy model we propose is based on multilayer Internet Protocol/Generalized Multi-Protocol Label Switching (IP/GMPLS) over optical networks. The control plane of the IP/GMPLS router is used to manage the power aware routing and traffic engineering for IP layer and optical network. It consists of dedicated channels to exchange messages and operates autonomously with data plane topology.

Figure 1 shows the overview of the IP/GMPLS based optical node. It consists of an IP/GMPLS router, Optical Cross-Connect (OXC), Erbium-Doped Fibre Amplifier (EDFA) for an intermediate in line amplification, and 40 channels based Wavelength Division Multiplexing (WDM) operating at $2.5 \mathrm{Gbps}$. For an energy purpose

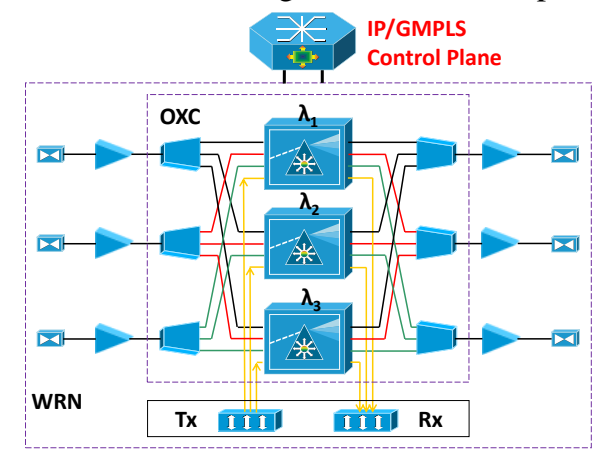

Figure 1: IP/GMPLS over optical network.

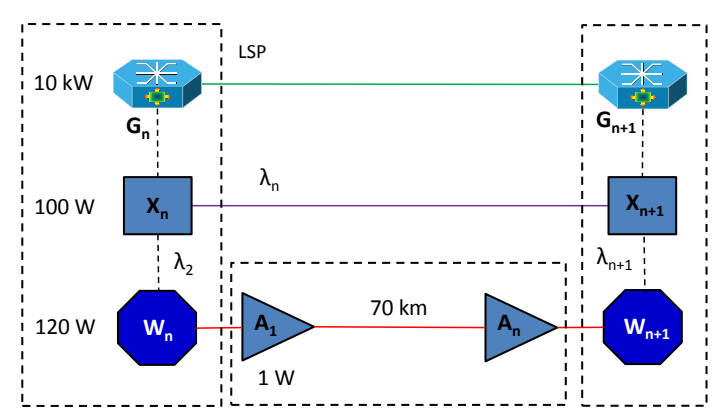

Figure 2: Green Photonic Network Model. 
calculations its equivalent configuration (see Figure 2) was used to form a network energy model to evaluate the IP/GMPLS power consumption and energy per bit based as illustrated in.

We assumed the network carrier bandwidth of OC-192 and average energy consumption of $1019 \mathrm{~nJ}$ per bit by following [2][3][6][7]; The $G_{n}$ denote IP/GMPLS dissipating power output of 10kW and energy consumption of $1000 \mathrm{~nJ}$ per a data bit. The $X_{n}$ represents OXC which is dissipating $100 \mathrm{~W}$ and consumes $10 \mathrm{~nJ}$ per bit. Whereas $W_{n}$ denote the WDM part of the node, with dissipating power of $120 \mathrm{~W}$ and energy consumption of $12 \mathrm{~nJ}$ per bit. The $A_{n}$ represents the EDFA amplifiers within WDM spans placed at every $70 \mathrm{~km}$ intervals. The power consumption is estimated to be $1 \mathrm{~W}$ with energy per a data bit of $0.1 \mathrm{~nJ}$.

\section{POWER CONSUMPTION}

We define the energy per bit consumed by a node as $\mathrm{E}_{\mathrm{b}}=\mathrm{P}_{\mathrm{T}} / \mathrm{C}$ where $\mathrm{P}_{\mathrm{T}}$ represents the total power consumption of the node; $\mathrm{C}$ is the bandwidth provided by the network link. The total power consumption of the node can be therefore expressed as

$$
\mathrm{P}_{\mathrm{T}}=\mathrm{P}_{\mathrm{g}}+\mathrm{P}_{\mathrm{x}}+\mathrm{P}_{\mathrm{a}}+\mathrm{P}_{\mathrm{w}}+\mathrm{P}_{\text {tr }}
$$

where $\mathrm{P}_{\mathrm{g}}, \mathrm{P}_{\mathrm{x}}, \mathrm{P}_{\mathrm{a}}, \mathrm{P}_{\mathrm{w}}$, and $\mathrm{P}_{\mathrm{tr}}$ represent power consumed by the IP/GMPLS router, Optical Cross-connect (OXC), EDFA, Wavelength Division Multiplexing (WDM) and transmitter, respectively.

\section{IMPLEMENTATION OF POWER AWARE IP/GMPLS OVER PHOTONIC NETWORKS}

For the purpose of this investigation the network energy model is based on a discrete-event simulation platform as describe in section 5. To achieve green network design with a desired power reduction the methodology we implemented is using "hibernation mode" approaches or "let sleep" the network equipment.

The hibernation mode as depicted in Figure 4 could be set up based on the following approaches:

1) Grouping the Nodes based on their activities and status. For example, Group 1 consists of Node 1 and Node 2 while Group 2 is Node 3, Node 4 and Node5.

2) Links(s) Segmentation. Any idled links identified by a Node will be shut down to conserve energy. For example, Segment 1 consists of Link 1, Link 2....Link n.

3) Partitioning the wavelength. To optimise energy efficiency, we teardown idle lightpaths within the Links and subgroup them. For instance, $\lambda_{1}, \lambda_{2}, \ldots . \lambda_{n}$ on Link 1.

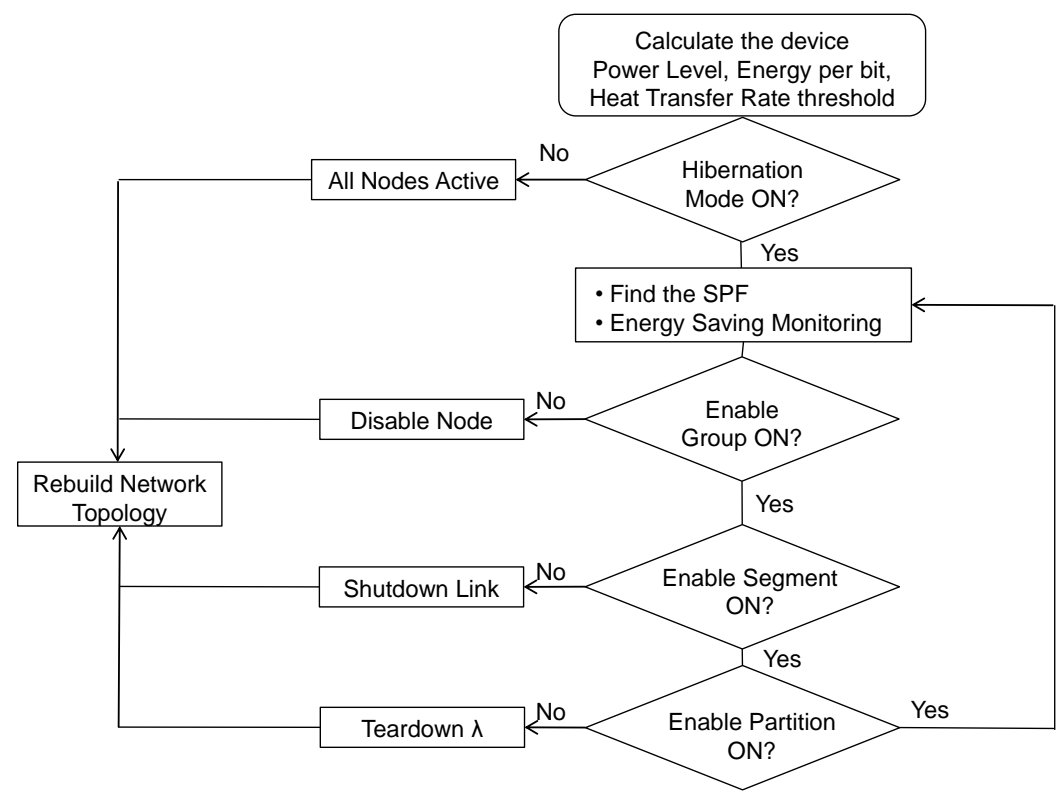

.Figure 3: A flowchart of Hibernation mode algorithm.

Hibernation mode $[\mathrm{H}]$ is define as collection of nodes subgroups, Links and wavelengths as

$$
H \epsilon \sum_{\forall n}\left[G_{n} \cup N_{n}\right]
$$

where $G_{n}=\left\{N_{1}, N_{2}, N_{3}, \ldots N_{n}\right\}$ represents given groups, $N_{n}=\left\{L_{1}, L_{2}, L_{3}, \ldots L_{n}\right\}$ nodes, $L_{n}=\left\{\lambda_{1}, \lambda_{2}, \lambda_{3}, \ldots \lambda_{n}\right\}$ Links, and $\lambda_{n}$ wavelengths, respectively.

The operation of the hibernation mode is described by a flowchart illustrated in Figure 4. Initially, the network equipment power consumption is computed based on 30\% (minimum), 50\% (medium) and 75\% (maximum) of 
energy saving levels, respectively. Then, the network measures the energy per bit and heat transfer rate levels. Now, if the network management prefers to enable the hibernation mode, three options can be executed based on the available network resources and a Shortest Path First (SPF) algorithm computation. If the network controller located in GMPLS control plane detects any idle nodes (no traffic activities), these nodes are grouped into a hibernation mode. The group configuration can be set (to OFF state) and system can immediately disable designated nodes. Whereby, when the node detects any traffic or becomes active, network controller may plan to reduce the energy consumption by trying to identify an idle link. This option is known as segment configuration. Here, the network resource computes any idle links to be shutdown. Once the shutdown links are determined, the network will be configured to aggregate the idle links which goes to the same node/destination. However, if the links are all active, the partition configuration can be triggered. In this process, algorithm will examine any idle wavelength $\left(\lambda_{n}\right)$ for teardown consideration. Nonetheless, if any of these conditions could not be fulfilled the process will be repeated and energy saving activities will be monitored again.

\subsection{Group and Segmentation}

Group and Segmentation could be ascertained to set up hibernation mode when network controller detects idle or inactive nodes. This method is exemplified in Figure 4 below. As illustrated in the diagram the IP/GMPLS nodes are denoted as A, B, C, D, E and Optical Nodes as 1, 2, 3, 4, 5, respectively. In a group configuration the active and inactive (idle) nodes are determined and categorized the following way: Group 1 consists of active nodes, namely Node 1 and Node 2 and is set up as "ON state" (active). Whilst Group 2 includes idle nodes Node 4 and Node 5 and is set up as "OFF state". However to fully optimize energy efficiency the segment configuration can be set to tear down any inactive links (ports) - in our example such idle nodes are in Group 1.

\subsection{Power aware Routing}

Figure 5 illustrates the network topology utilised by the National Science Foundation (NSFnet) network. The IP/GMPLS nodes are linked by a bidirectional pair of single mode fibres. The node functions include maintaining information on total power consumption and energy per bit levels. Furthermore, the node Data

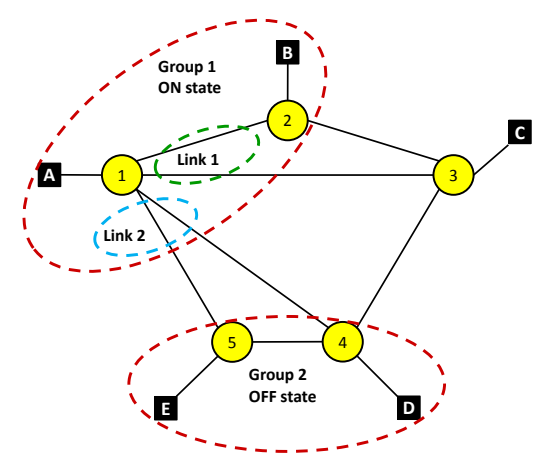

Figure 4: Group / segment configuration.

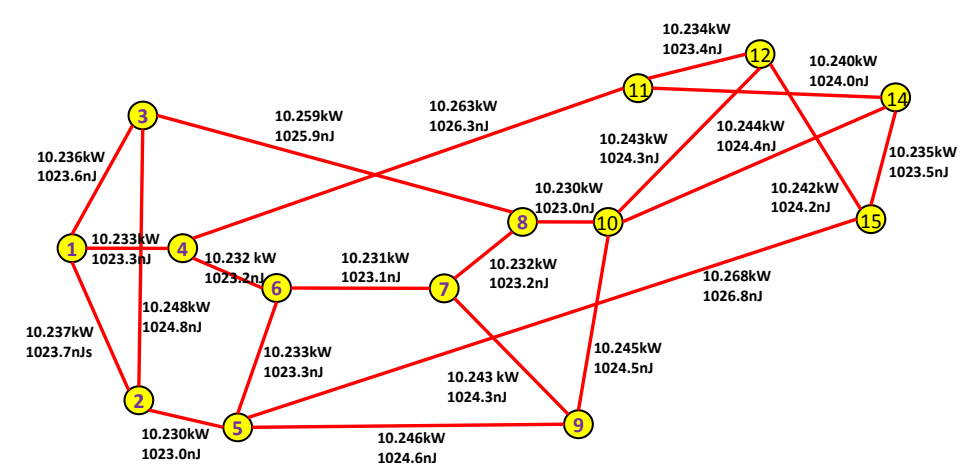

Figure 5: The NSFnet Network Topology.

Tables are responsible to sustain updated information on the following; (1) Network Topology Table; (2) Routing and Wavelength Assignment Table; (3) Lightpaths Information Table; (4) Forwarding Table. Wherein, the standard GMPLS signalling and routing protocols are implemented based on Internet Engineering Task Force (IETF) standard draft $[9,10]$.

The power aware routing algorithm is based on power consumed by each node and a shortest path first (SPF) approach as determined by algorithm in Figure 3. The algorithm tries to put as many links and nodes as possible into a hibernation mode [5]. This could be done by grouping and segmentation as explained in the previous section. During this hibernation process, the nodes advertise their routing and data tables information status to the control plane to constantly update power consumption within network. Meanwhile Hibernation configuration must keep Erbium-Doped Fibre Amplifiers (EDFA) always switched ON and nodes in minimum operation mode.

\section{SIMULATION RESULTS}

The Hibernation approach on group and segment configuration is executed to test the IP/GMPLS over optical networks to measure power consumption. The performance metrics involved takes into an account the average power consumption, blocking probability and average request blocking [3-4], [8]. The blocking probability represents the ratio of the number of discarded links over the number of requested connections in the network. The total number of connection requests that are available to be routed is known as offered load (traffic load) denoted in Erlangs. It is assumed that all links are equal in terms of number of wavelength (eight), that the message length is fixed at 256 bytes, and the nodal process delay is $20 \mathrm{~ms}$. 
Our simulation work is based on the discrete event model tool known as OMNet++ (Object Modular Network Tested in $\mathrm{C}++$ ). The network topology used in our simulation is the National Science Foundation (NSFnet) network shown in Fig. 5; the bandwidth is OC-192 with bidirectional single mode fibre links implemented. For the purpose of this simulation and ease of calculation the EDFA amplifiers bear minimum power consumption of $1 \mathrm{~W}$ and are placed every $70 \mathrm{~km}$.

The advantage of grouping IP/GMPLS over optical network topology has been verified on NSFnet depicted in Figure 5 by setting up groups which are define as follows: $G_{1}=[1,2,3,4,5], G_{2}=[6,7,8,9]$, and $G_{3}=$ $[10,11,12,13,14]$ based on multilayer power aware routing algorithm. The group and segment configuration has been set to allow hibernation on the designated nodes and links. Hibernation mode is in use when groups and segments are set to OFF state (nodes disabled). These conditions are determined by observing whether nodes and links are in an idle state or whether there is any active traffic in the given part of the network.

Figure 6 illustrates results for NSFnet when the Network topology is configured to be in hibernation mode. Power consumption decreases about $1 \%$ with each decrease of offered network traffic load by way of setting OFF individual Groups.

Figures 7 present the effect of the hibernation mode on average wavelength request loss during group set into OFF state. We observe that whenever the energy decreases, the probability of blocking increases about $70 \%$ due to wavelength unavailability or by the node being in hibernation state.

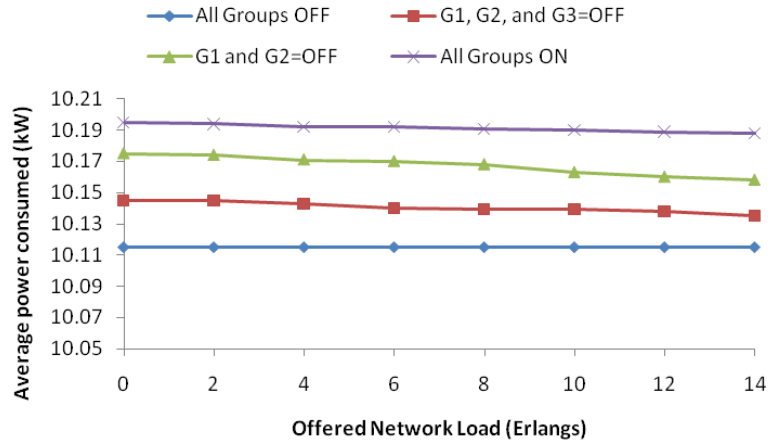

Figure 6: Averaged Power consumed for NSFnet.

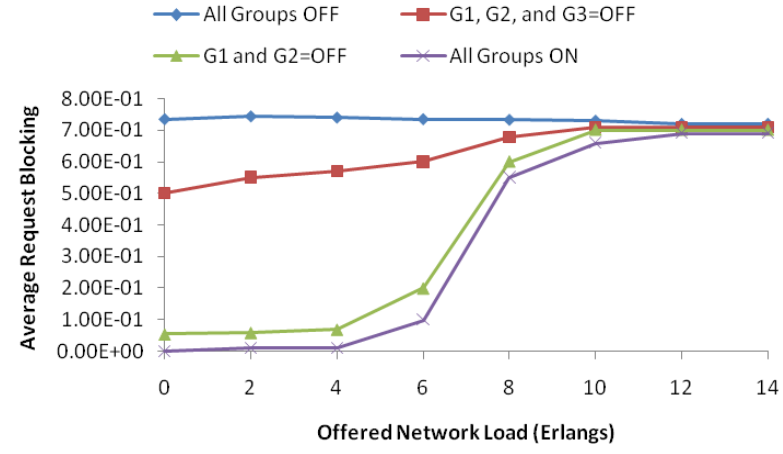

Figure 7: Request blocking for NSFnet.

\section{CONCLUSIONS}

The paper presents an approach to energy saving in multilayer routing for IP/GMPLS based optical networks. We examined the impact of hibernation mode on selective groups and calculated the average wavelength request loss as well as energy per bit consumed by IP/optical networks. Our results shows that by applying hibernation mode on energy aware routing algorithm and selective groups, a significant amount of energy can be saved in IP/optical networks. In addition optical technologies leading to energy savings in optical networks also needs very serious consideration.

\section{REFERENCES}

[1] M.S. Lebby, Green Photonics Technology and Markets, IET Electronic Letters, vol. 45. pp. 1007-1007, Sept. 2009.

[2] J. Balinga, et al.: Energy Consumption in Optical IP Networks, IEEE Journal of Lightwave Technology, vol. 27. pp. 2391-2403, July 2009.

[3] B.G. Bathula, et al.: Energy Efficient Architectures for Optical Networks, in Proc. London Communications Symposium (LCS), London, UK, Sep. 2009.

[4] J. Chabarek, et al:: Power Awareness in Network Design and Routing, in IEEE Proc. $27^{\text {th }}$ Conference on Computer Communications INFOCOM 2008, Phoenix, USA, pp. 1130-1138, April 2008.

[5] N. Vasic, et al:: Energy-Aware Traffic Engineering, EPFL Technical Report NSL-REPORT-2008-004, Switzerland, 2008.

[6] Cisco System Data Sheets, [Online]. Available: http://www.cisco.com

[7] MRV Communications Inc Data Sheet, [Online]. Available: http://www.mrv.com

[8] S. Albarrak, et al.: Pre-Allocated Restoration Techniques in a Distributed GMPLS-Based Mesh Optical Network, $4^{\text {th }}$ International Conference Sciences of Electronic, Technologies of Information and Telecommunications, SETIT 2007, Tunisia, March 2007.

[9] L. Berger, Generalized Multi-Protocol Label Switching (GMPLS) Signalling Functional Description, [Online]. Available: http://www.faqs.org/ftp/rfc/pdf/rfc3471.txt.pdf, Jan. 2003.

[10] J. Lang, Generalized Multi-Protocol Label Switching (GMPLS) Signalling Resource Reservation ProtocolTraffic Engineering (RSVP-TE) Extension, [Online]. Available at: http://www.faqs.org/rfcs/rfc4208.html. 